MATHEMATICAL PROGRAMS WITH A TWO-DIMENSIONAL REVERSE CONVEX CONSTRAINT

P.T. Thach, R.E. Burkard

Technische Universität Graz

and

W. Oettli

Universität Mannheim

Nr. 113 (1990) 


\title{
MATHEMATICAL PROGRAMS WITH A TWO-DIMENSIONAL REVERSE CONVEX CONSTRAINT
}

\author{
P.T. Thach ${ }^{1}$, R.E. Burkard \\ Technische Universität Graz, $8010 \mathrm{Graz}$, Austria \\ and \\ W. Oettli \\ Universität Mannheim, 6800 Mannheim, Germany
}

\begin{abstract}
We consider the problem $\min \{f(x): x \in G, T(x) \notin$ int $D\}$, where $f$ is a lower semicontinuous function, $G$ a compact, nonempty set in $\mathbb{R}^{n}, D$ a closed convex set in $\mathbb{R}^{2}$ with nonempty interior, and $T$ a continuous mapping from $\mathbb{R}^{n}$ to $\mathbb{R}^{2}$. The constraint $T(x) \notin$ int $D$ is a reverse convex constraint, so the feasible domain may be disconnected even when $f, T$ are affine and $G$ is a polytope. We show that this problem can be reduced to a quasiconcave minimization problem over a compact convex set in $\mathbb{R}^{2}$, and hence can be solved effectively provided $f, T$ are convex and $G$ is convex or discrete. In particular, we discuss a reverse convex constraint of the form $\langle c, x\rangle \cdot\langle d, x\rangle \leq 1$. We also compare the approach in this paper with the parametric approach.
\end{abstract}

Key words. Reverse convex program, global optimization.

\section{Introduction}

In recent years, convex programs with an additional reverse convex constraint have attracted attention of several researchers (see e.g. Hillestad \& Jacobsen [34], Singer [14], Tuy [21], Tuy \& Thuong [22-23], Thach [16], Muu [11], Thoai [19], Fülöp [2]). A general form of this problem is the following:

1 On leave from Institute of Mathematics, Hanoi 


$$
\begin{aligned}
& \text { minimize } f(x) \\
& \text { subject to } x \in G \\
& \text { and } x \notin \text { int } \Delta,
\end{aligned}
$$

where $f$ is a convex function, $G$ and $\Delta$ are convex sets. The difficulty of problem (1.1)-(1.3) comes from constraint (1.3) which is called a reverse convex constraint. Without (1.3) the problem is an ordinary convex program. Problem (1.1)-(1.3) belongs to a class of hard optimization problems. Indeed, if $f$ is a constant function, $\Delta$ the unit ball in $\mathbb{R}^{n}$ and $G$ a polytope defined by a finite number of linear inequalities, then problem (1.1)-(1.3) becomes a set containment problem which has been shown to be NP-complete (see e.g. Freund [1]). Tuy [21] reduced this problem under a stability condition to a sequence of linearly constrained concave minimization problems in $\mathbb{R}^{n}$. Up to now, the size of reverse convex programs which can be solved to optimality is very limited.

In this paper we investigate a special class of reverse convex programs where $G$ is an arbitrary compact, nonempty set in $\mathbb{R}^{n}$, and the reverse convex constraint has the form

$$
T(x)=\left(T_{1}(x), T_{2}(x)\right) \notin \operatorname{int} D,
$$

where $D$ is a closed convex set in $\mathbb{R}^{2}$ and $T=\left(T_{1}, T_{2}\right)$ a continuous mapping from $\mathbb{R}^{n}$ to $\mathbb{R}^{2}$. Our purpose is to show that, if we have available an efficient algorithm to solve the problem

$$
\min \left\{f(x): x \in G, t_{1} T_{1}(x)+t_{2} T_{2}(x) \geq \alpha\right\}
$$

then we have an efficient algorithm to solve problem (1.1), (1.2) and (1.4) as well. In many cases problem (1.5) is easy to solve, for example, if $T_{1}, T_{2}$ are linear functions, $f$ a convex function and $G$ a convex set. This case includes the problem

$$
\min \{f(x): x \in G,\langle c, x\rangle \cdot\langle d, x\rangle \leq 1\}
$$


where $f$ is convex, $G$ is a convex set in $\mathbb{R}_{+}^{n}$, and $c$ and $d$ are vectors in $I R_{+}^{n}$. Indeod, by setting $T_{1}(x)=\langle c, x\rangle, T_{2}(x)=\langle d, x\rangle$, and

$$
D=\left\{v=\left(v_{1}, v_{2}\right) \in \mathbb{R} R_{+}^{2}: v_{1} \cdot v_{2} \geq 1\right\}
$$

we can convert problem (1.6) into problem (1.1), (1.2) and (1.4). The product of two linear functions appears in some applications as VLSI chip design, transportation or micro economics. Optimization problems dealing with it have been considered by several authors (see e.g. Pardalos [13], Konno et al. [9-10], Suzuki et al. [15]). We shall deal with this particular problem in Section 4. Another example where $f, T_{1}, T_{2}$ are linear functions and $G=\{0,1\}^{n}$ leads to the $0-1$ knapsack problem and can be solved by a pseudopolynomial algorithm (see e.g. [12]).

In order to solve problem (1.1), (1.2) and (1.4), we reduce it to a quasiconcave minimization problem over a closed convex set in $\mathbb{R}^{2}$. By using a cutting plane approach for the reduced problem we obtain an effective algorithm for the problem under investigation.

This paper consists of five sections. In Section 2 we reduce problem (1.1), (1.2) and (1.4) to a quasiconcave minimization problem in $\mathbb{R}^{2}$. In Section 3 we present a solution method. In Section 4 we specialize the reverse convex constraint to a product of two linear functions and illustrate the developed method by a numerical example. Finally, we draw some conclusions in Section 5.

\section{Reduction to a quasiconcave minimization problem in the plane}

Let us assume that $G$ is a compact, nonempty set in $\mathbb{R}^{n}, D \subseteq \mathbb{R}^{2}$ is a closed convex set with int $D \neq \emptyset, f: G \longrightarrow \mathbb{R}$ is a lower semicontinuous (lsc) function, and $T$ a continuous mapping from $\mathbb{R}^{n}$ to $\mathbb{R}^{2}$. The problem under consideration has the form

$$
\min \{f(x): x \in G, T(x) \notin \operatorname{int} D\}
$$


We assume that $(P)$ has at least one feasible solution. Let $w$ be a minimizer of $f$ on $G$. If $T(u)$ int $D$, then $w$ solves $(P)$, and we terminate. Therefore we morict out attention in the following to the case $T(w)$ Ent $D$.

Let $V:=D-T(w)$. Since $T(w) \in$ int $D, V$ contains 0 in its interior. Denote by $E$ the polar set of $V$ in $\mathbb{R}^{2}$ :

$$
E:=\left\{t \in \mathbb{R}^{2}:\langle t, v\rangle \leq 1 \forall v \in V\right\} .
$$

$V$ is a neighborhood of 0 , therefore the polar $E$ is a nonempty compact, convex set. We define $h: \mathbb{R}^{2} \longrightarrow \mathbb{R} \cup\{+\infty\}$ by

$$
h(t):=\inf \{f(x): x \in G,\langle t, T(x)-T(w)\rangle \geq 1\} .
$$

Let $0 \leq \lambda \leq 1$. Then from

$$
\begin{gathered}
\left\{x:\left\langle\lambda t^{1}+(1-\lambda) t^{2}, T(x)-T(w)\right\rangle \geq 1\right\} \\
\subseteq\left\{x:\left\langle t^{1}, T(x)-T(w)\right\rangle \geq 1\right\} \cup\left\{x:\left\langle t^{2}, T(x)-T(w)\right\rangle \geq 1\right\}
\end{gathered}
$$

follows

$$
h\left(\lambda t^{1}+(1-\lambda) t^{2}\right) \geq \min \left\{h\left(t^{1}\right), h\left(t^{2}\right)\right\}
$$

Hence $h$ is quasiconcave. Moreover $h$ is lsc from the compactness of $G$, the continuity of $T$ and the lower semi-continuity of $f$.

We are now going to show that the original problem $(P)$ can be reduced to minimizing $h$ over the polar set $E$.

We have

$$
\begin{gathered}
T(x) \notin \operatorname{int} D \Longleftrightarrow T(x)-T(w) \notin \operatorname{int} V, \\
T(x)-T(w) \notin \text { int } V \Longleftrightarrow(\exists t \in E:\langle t, T(x)-T(w)\rangle \geq 1) .
\end{gathered}
$$

\section{Therefore}

$$
\begin{aligned}
& \inf \{f(x): x \in G, T(x) \notin \operatorname{int} D\} \\
= & \inf \{f(x): x \in G, T(x)-T(w) \notin \operatorname{int} V\} \\
= & \inf \{f(x): x \in G, t \in E,\langle t, T(x)-T(w)\rangle \geq 1\} \\
= & \inf _{t \in E} \inf _{x}\{f(x): x \in G,\langle t, T(x)-T(w)\rangle \geq 1\} \\
= & \inf \{h(t): t \in E\} .
\end{aligned}
$$


Therefore we consider the problem

$$
\min \{h(t): t \in E\}
$$

and for every $t \in \mathbb{R}^{2}$ the problem

$$
\inf \{f(x): x \in G,\langle t, T(x)-T(w)\rangle \geq 1\}
$$

Then $h(t)=\inf \left(L_{t}\right)$. Since $h$ is lsc and $E$ is compact, $(\tilde{P})$ has an optimal solution. Thus we have shown

\section{Theorem 2.1.}

i) Problem $(\widetilde{P})$ has an optimal solution;

ii) $\inf (P)=\inf (\widetilde{P})$;

iii) If $t^{*}$ is an optimal solution of $(\widetilde{P})$ and $x^{*}$ solves $\left(L_{t^{*}}\right)$, then $x^{*}$ solves $(P)$.

\section{A solution method}

Let us first introduce concepts of approximate solutions. Let $\left\{D_{\varepsilon}, \varepsilon \geq 0\right\}$ be a family of subsets in $\mathbb{R}^{2}$ such that $D_{0}=$ int $D$ and, for $\varepsilon>0, D_{\varepsilon}$ is a closed subset of int $D$. The constraint $T(x) \notin D_{\varepsilon}$ is then a relaxation of $T(x) \notin$ int $D$.

\section{Definition 3.1.}

i) $x$ is called an $\eta$-optimal solution $(\eta \geq 0)$ of $\left(L_{t}\right)$, iff $x$ is feasible for $\left(L_{t}\right)$ and $f(x) \leq \inf \left(L_{t}\right)+\eta$.

ii) $x$ is called $(\eta, \varepsilon)$-optimal, iff $x \in G, T(x) \notin D_{\varepsilon}$ and $f(x) \leq \inf (P)+\eta$.

Now we present an algorithm for finding an $(\eta, \varepsilon)$-optimal solution of $(P)$, which essentially is the outer approximation method specialized for $(\widetilde{P})$.

\section{Algorithm 3.1.}

\section{Initialization.}

Let $W_{0} \subseteq \mathbb{R}^{2}$ be a triangle containing the compact convex set $E$. Let $U_{-1}:=\emptyset$. Set $k:=0$. Enter Iteration $k$. 


\section{Iteration $\mathrm{k}$.}

Let $l_{k}:=v e r$ tex set of $W_{k}$. For all $t \in U_{k} \backslash U_{k-1}$ determine $x^{*}(t)$ as an $\eta$-optimal solution of $\left(L_{i}\right)$. [ff $\left(L_{t}\right)$ s not feasble, then aet hetionsly $\left.f\left(t^{*}(b)\right):=+\infty\right]$.

Step k.a.

Let $t^{k} \in \operatorname{argmin}\left\{f\left(x^{*}(t)\right): t \in U_{k}\right\}$, and $x^{k}:=x^{*}\left(t^{k}\right)$. Go to Step k.b.

Step k.b.

If $T\left(x^{k}\right) \notin D_{\varepsilon}$, then terminate: $x^{k}$ is $(\eta, \varepsilon)$-optimal (see the comment below). Otherwise, go to Step k.c.

Step k.c.

Let $W_{k+1}:=W_{k} \cap\left\{t: \dot{l}_{k}(t) \leq 0\right\}$, where $l_{k}$ is an affine function such that $l_{k}\left(t^{k}\right)>0$ and $l_{k}(t) \leq 0$ for all $t \in E$. Go to iteration $\mathrm{k}+1$.

\section{Comments}

Since $x^{*}(t)$ is an $\eta$-optimal solution of $\left(L_{t}\right)$ one has

$$
h(t) \leq f\left(x^{*}(t)\right) \leq h(t)+\eta
$$

Since $x^{k}$ is feasible for $\left(L_{t^{k}}\right)$ one has

$$
x^{k} \in G \text { and }\left\langle t^{k}, T\left(x^{k}\right)-T(w)\right\rangle \geq 1
$$

Since $h$ is quasiconcave one has

$$
\min \left\{h(t): t \in W_{k}\right\}=\min \left\{h(t): t \in U_{k}\right\}
$$

Since $E \subseteq W_{k}$ for any $\mathrm{k}$, one obtains

$$
\begin{aligned}
\min (P) & =\min \{h(t): t \in E\} \\
& \geq \min \left\{h(t): t \in W_{k}\right\} \\
& =\min \left\{h(t): t \in U_{k}\right\} \\
& \geq \min \left\{f\left(x^{*}(t)\right)-\eta: t \in U_{k}\right\}=f\left(x^{k}\right)-\eta .
\end{aligned}
$$

If termination occurs in iteration $\mathrm{k}$, then the solution $x^{k}$ is $(\eta, \varepsilon)$-optimal, since $T\left(x^{k}\right) \notin D_{\varepsilon}$ and $f\left(x^{k}\right) \leq \min (P)+\eta$ from (3.1). 
If no termination occurs in iteration $\mathrm{k}$, then $t^{k} \notin E$. Suppose the contrary $t^{k} \in E$. Together with $\left(t^{k}, T\left(x^{k}\right)-T(w)\right) \geq 1$ this imples $T\left(x^{k}\right)-T(w) \notin$ int $V$ and herofe $T\left(x^{k}\right)$ int $D$. But then $T\left(a^{k}\right) D_{s}$, and temination would occur. Thus, if no termination occurs in iteration $\mathrm{k}$, then there exists an affine function $l_{k}$, which cuts the vertex $t^{k}$ from $E$. Indeed, from $t^{k} \notin E$ and from the definition of $E$ follows $\sup _{v \in V}\left\langle t^{k}, v\right\rangle>1$. Hence there exists a $v^{k} \in V$ such that $\left\langle t^{k}, v^{k}\right\rangle>1$. Now the affine function $l_{k}(t):=\left\langle t, v^{k}\right\rangle-1$ satisfies $l_{k}\left(t^{k}\right)>0$, while $l_{k}(t) \leq 0$ for all $t \in E$. Thus the function $l_{k}$ cuts the vertex $t^{k}$ from $E$.

$W_{0}$ is a triangle. Since $W_{k}$ is obtained from $W_{k-1}$ by adding a linear constraint, $U_{k}$ can easily be computed by using the information of $U_{k-1}$. Furthermore, for $k>0, U_{k} \backslash U_{k-1}$ contains at most two points. So, in each iteration $\mathrm{k}$ we have to solve at most two subproblems $\left(L_{t}\right)$.

We discuss now the finiteness of the algorithm.

Definition 3.2. We say that the sequence of cutting functions $l_{k}$ used in Algorithm 3.1 is convergent, if every cluster point of the sequence $\left\{t^{k}\right\}$ belongs to E.

We can find sufficient conditions for the convergence of the sequence of cutting functions in e.g. Kleibohm [8] and Tuy [20].

Theorem 3.1. If the sequence of cutting functions $l_{k}$ is convergent, then Algorithm 3.1 terminates after finitely many iterations for every $\varepsilon>0$.

Proof. Assume that no termination occurs. Since $\left\{t^{k}\right\} \subseteq W_{0}$ and $W_{0}$ is compact, there exists a cluster point $t^{*}$ of $\left\{t^{k}\right\}$. Since $\left\{x^{k}\right\} \subseteq G, G$ compact, there exist subsequences $t^{k_{\mu}} \longrightarrow t^{*}, x^{k_{\mu}} \longrightarrow x^{*} \in G$. It follows that $\left\langle t^{*}, T\left(x^{*}\right)-\right.$ $T(w)\rangle \geq 1$. The convergence of the cutting functions yields $t^{*} \in E$. From (2.4) and (2.5) follows then $T\left(x^{*}\right) \notin$ int $D$, hence $T\left(x^{*}\right) \notin D_{\varepsilon}$. Since $D_{\varepsilon}$ is closed, this implies $T\left(x^{k_{\mu}}\right) \notin D_{\varepsilon}$ for all $k_{\mu}$ sufficiently large. But this contradicts non-termination. 


\section{Remark 3.1}

The same algorith can be used to solve problems of the form

$$
\min _{x, i}\{f(x): x \in G, t \in E, W(x, t) \geq 0\}
$$

where $\Psi(x, \cdot)$ is quasiconvex and $E$ is a compact, convex set in $\mathbb{R}^{2}$. In this case the function

$$
h(t):=\inf \{f(x): x \in G, \Psi(x, t) \geq 0\}
$$

is quasiconcave. The problems

$$
\inf \{f(x): x \in G, \Psi(x, t) \geq 0\}
$$

are convex, if $f, G$ are convex and $\Psi(\cdot, t)$ is quasiconcave.

\section{Reverse convex programs dealing with the product of two linear} functions

In this section we deal with the important special case of $(P)$, where the reverse convex constraint can be written in the form

$$
\langle c, x\rangle \cdot\langle d, x\rangle \leq 1
$$

for two linearly independent vectors $c$ and $d \in \mathbb{R}_{+}^{n}$. In this case

$$
\left(T_{1}(x), T_{2}(x)\right)=(\langle c, x\rangle,\langle d, x\rangle), \text { and } D=\left\{v \in \mathbb{R}_{+}^{2}: v_{1} \cdot v_{2} \geq 1\right\}
$$

In principle, this problem can be written in the form:

$$
\operatorname{minimize} F(\xi) \text { s.t. } 0<\xi<\infty
$$

with

$$
F(\xi)=\inf \left\{f(x): x \in G,\langle c, x\rangle \leq \xi,\langle d, x\rangle \leq \frac{1}{\xi}\right\}
$$

The difficulty of this approach however consists in the complicated behavior of the function $F$, implicitly defined via (4.2). For example, the function $F$ may 
have an infinite number of local optima (see the Example 4.1). Therefore we coeralize the method developed in the previous sections to this situation.

Th the bilowing we deat wh

$$
\min f(x) \text { s.t. } x \in G \text { and }\langle c, x\rangle \cdot\langle d, x\rangle \leq 1 \text {, }
$$

where we assume that $f$ is lower semicontinuous and $G$ is a compact, nonempty set in $\mathbb{R}_{+}^{n}$. Let $w$ be a solution of $\min \{f(x): x \in G\}$. If $w$ satisfies the constraint (4.1), then $w$ solves problem (4.3), too. In this case the reverse convex constraint is not essential. So, we restrict our attention to the case where $\langle c, w\rangle \cdot\langle d, w\rangle>1$. We can explicitly determine the polar set $E$ by the following lemma.

Lem ma 4.1. The polar set $E$ of $V$ has the form

$$
E=\left\{\left(t_{1}, t_{2}\right):-t_{1}\langle c, w\rangle-t_{2}\langle d, w\rangle-2\left(t_{1} t_{2}\right)^{\frac{1}{2}} \leq 1, t_{1} \leq 0, t_{2} \leq 0\right\}
$$

From the lemma we can easily construct a triangle $W_{0} \supseteq E$ such that

$$
W_{0} \subseteq \mathbb{R}_{-}^{2}
$$

and a cutting function separating a given point outside $E$ from $E$. Set for $\varepsilon>0$

$$
D_{\varepsilon}=\left\{v=\left(v_{1}, v_{2}\right): v_{1} \cdot v_{2} \geq 1+\varepsilon, v_{1} \geq 0, v_{2} \geq 0\right\}
$$

It is obvious that $D_{\varepsilon}$ is closed and $D_{\varepsilon} \subseteq$ int $D$.

For any $\eta>0, \varepsilon>0$ Algorithm 3.1 yields an $(\eta, \varepsilon)$-optimal solution after finitely many iterations.

\section{Example 4.1}

Let us illustrate the foregoing ideas by the following problem in $\mathbb{R}^{2}$.

$$
\begin{gathered}
\text { Minimize }-x-y, \text { subject to } \\
2^{2 k-1} x+\left(1.1 \cdot 2^{k+1}-1\right)\left(1.1 \cdot 2^{k}-1\right) y \geq 2^{k-1}, \forall k=0,1,2, \cdots
\end{gathered}
$$




$$
\begin{gathered}
0.2 \leq x \leq 2.2,0.4 \leq y \leq 5, \\
x \cdot y \leq 1
\end{gathered}
$$

In this example, $G$ detined by (4.7)-(1.8) is a compact, convex set in $i R_{+}^{2}$. For each $\xi>0$ set

$$
F(\xi)=\inf \left\{-x-y:(x, y) \in G, x \leq \xi, y \leq \frac{1}{\xi}\right\}
$$

Then the function $F$ has an infinite number of local optima. The set of local optima of $F$ in the segment $[0.2,2.2]$ is

$$
2.2-\frac{1}{2^{k-1}}, k=0,1,2, \cdots
$$

but $F$ has only one global minimizer at $\xi=0.2$. It can easily be seen that $(x, y)=(2.2,5)$ is a minimizer of $(4.6)-(4.8)$. Therefore, $w=(2.2,5)$ and

$$
E=\left\{\left(t_{1}, t_{2}\right):-2.2 \cdot t_{1}-5 \cdot t_{2}-2\left(t_{1} t_{2}\right)^{\frac{1}{2}} \leq 1, t_{1} \leq 0, t_{2} \leq 0\right\}
$$

(see Fig.1).

Now; let us apply Algorithm 3.1 with $\varepsilon=0, \eta=0$ to solve (4.6)-(4.9).

Initialization. Let $W_{0}$ be a triangle defined by the intersection of $\mathbb{R}_{-}^{2}$ and the half space supporting $E$ at $(-1 / 5.2,-1 / 5.2)$.

Then, $U_{0}=\{(0,0),(0,-0.25),(-1 / 1.2,0)\}$; 
$x^{*}(0,0)=\emptyset, h(0,0)=\infty ;$

$\pi^{*}(0, \cdots-2.25)=(2.2 .1), h(0,-0.25)=-3.2$

$x^{*}(-1,2,0)=(1,5) h(-1 / 1,2,0)=-0$.

Iteration 0 .

Step 0.a. $t^{0}=(-1 / 1.2,0)$.

Step 0.b. $\left\langle c, x^{*}\left(t^{0}\right)\right\rangle \cdot\left\langle d, x^{*}\left(t^{0}\right)\right\rangle=5>1$.

Step 0.c. We choose the cutting line

$\alpha^{0} t_{1}+\beta^{0} t_{2}+\gamma^{0}=0$ with $\alpha^{0}=-1, \beta^{0}=0, \gamma^{0}=-0.5$ (see Fig.1)

and get as new vertices:

$U_{1} \backslash U_{0}=\{(-0.5,-0.1),(-0.5,0)\}$;

$x^{*}(-0.5,-0.1)=(0.2,5), h(-0.5,-0.1)=-5.2$;

$x^{*}(-0.5,0)=(0.2,5), h(-0.5,0)=-5.2$;

Iteration 1.

Step 1.a. $t^{1}=(-0.5,0)$;

Step 1.b. $\left\langle c, x^{*}\left(t^{1}\right)\right\rangle \cdot\left\langle d, x^{*}\left(t^{1}\right)\right\rangle=1$ : STOP with the optimal solution $(0.2,5)$.

\section{Discussion}

For the perfomance of Algorithm 3.1 we need an available efficient algorithm for the subproblem $\left(L_{t}\right)$. In the algorithm we are, in fact, considering subproblems with only $t \in W_{0}$. So, an available efficient algorithm for $\left(L_{t}\right)$ is required with only $t \in W_{0}$. In the case, where $D=\left\{\left(v_{1}, v_{2}\right): v_{1} v_{2} \geq 1, v_{1} \geq 0, v_{2} \geq 0\right\}$, by virtue of (4.5) we can construct $W_{0} \subseteq \mathbb{R}_{-}^{2}$. Therefore we need an efficient algorithm for $\left(L_{t}\right)$ with $t \leq 0$. If $f, T_{1}, T_{2}$ are convex and $G$ is a convex set, then $\left(L_{t}\right)$ for $t \leq 0$ is an ordinary convex program. Thus, we also obtain an efficient method for the problem

$$
\min \left\{f(x): x \in G, T_{1}(x) \cdot T_{2}(x) \leq 1\right\}
$$

where $T_{1}, T_{2}$ are nonnegative-valued convex functions. 
Since $h$ is quasiconcave, a minimizer of $h$ on $E$ must be located at at least one extreme point of $E$. Becanse in the plane the set of extreme points of a closed conves boty is contained in the boundary curve, let g be the restriction of h to the boundary curve $C$ of $E$. Then problem $(P)$ can be reduced to the problem of minimizing $g$ on $C$. This is another parametric approach for solving $(P)$. As mentioned in the introduction and shown in the Example 4.1, the function $g$ may have infinitely many local optima on $C$.

A duality between Quasiconvex Minimization over the complement of a convex set and Quasiconvex Maximization over a convex set has recently been established by one of the authors (see $[17,18]$ ). If we consider problem $(P)$ as a primal problem, then problem $(\tilde{P})$ can be considered as its dual. Thus in this case we see that the dual problem is much easier than the primal. The approach presented in this paper could also be extended for cases where the dimension $\mathrm{k}$ of $D$ is greater than 2. In such cases the dual program is a concave program of more than two variables, but it is still simpler than the primal, if the dimension of the reverse convex constraint is much smaller than $n$. In that case, we can use available algorithms for linearly constrained quasiconcave minimization subproblems of $\mathrm{k}$ dimensions at each iteration (see Horst and Tuy [7]).

The algorithm presented in Section 3 is an outer approximation. Recently, branch and bound methods (see e.g. Horst [6], Horst and Tuy [7], Tuy and Horst [24]) became practicable for Quasiconvex Maximization. We just indicate such an approach and leave the detailed development of such an algorithm for $(\widetilde{P})$ to a separate work.

\section{Acknowledgment}

The partial support by the Austrian Science Foundation, Project S 32/01 is gratefully acknowledged. 


\section{References}

[1] Frend, R.M. and J.N. Orlin (1985), On the complexity of four set connament problems. Wathemaical Programming $33,139-45$.

[2] Füiöp, J. (1988), A finite cuting plane method for solving inear programs with an additional reverse convex constraint. Working Paper MO/73, MTA SZTAKI, Hungarian Academy of Sciences.

[3] Hillestad, R.J. and S.E. Jacobsen (1980), Linear programs with an additional reverse convex constraint. Applied Mathematics and Optimization 6, 257-269.

[4] Hillestad, R.J. and S.E. Jacobsen (1980), Reverse convex programming. Applied Mathematics and Optimization 6, 63-78.

[5] Horst, R. (1988), Deterministic global optimization with partition sets whose feasibility is not known. Application to concave minimization, reverse convex constraints, d.c. programming and Lipschitzian optimization. Journal of Optimization Theory and Applications 58, 11-37.

[6] Horst, R. (1990), Deterministic methods in constrained global optimization: some recent advances and new fields of application. Naval Research Logistics Quarterly 37, 433-471.

[7] Horst, R. and H. Tuy (1990), Global Optimization (Deterministic Approaches). Springer, Berlin.

[8] Kleibohm, K. (1966), Ein Verfahren zur approximativen Lösung von konvexen Programmen. Dissertation, Universität Zürich.

[9] Konno, H. and T. Kuno (1989), Linear multiplicative programming. Preprint, Tokyo Institute of Technology.

[10] Konno, H. and T. Kuno (1989), Generalized linear multiplicative and fractional programming. Preprint, Tokyo Institute of Technology.

[11] Muu, L.D. (1985), A convergent algorithm for solving linear programs with an additional reverse convex constraint. Kybernetika 21, 428-435.

[12] Nemhauser, G.L. and L.A. Wolsey (1988), Integer and Combinatorial Op- 
timization. J. Wiley and Sons, New York.

[13] Pardalos, P.M. (1988), On the global minimization of the product of two linear functions over a polytope . Preprint, Pennsylvania State University; presented at the 13-th International Symposium on Mathematical Programming, Tokyo, 1988.

[14] Singer, I. (1980), Minimization of continuous convex functionals on complements of convex subsets of locally convex spaces. Optimization 11, 221-234.

[15] Suzuki, S., P.T. Thach and T. Tanaka (1989), On methods for minimizing the product of two convex functions. Preprint, Hanoi Institute of Mathematics.

[16] Thach, P.T. (1985), Convex programs with several additional reverse convex constraints. Acta Mathematica Vietnamica 10, 35-57.

[17] Thach, P.T. (1989), Quasiconjugates of functions, duality relationship between quasiconvex minimization under a reverse convex constraint and quasiconvex maximization under a convex constraint, and applications. Preprint n.152, Institute of Mathematics, Graz University of Technology.

[18] Thach, P.T. (1989), Dual problem for reverse convex programs. Preprint, Hanoi Institute of Mathematics.

[19] Thoai, N.V. (1988), A modified version of Tuy's method for solving d.c. programming problems. Optimization 19, 665-674.

[20] Tuy, H. (1983), On outer approximation methods for solving concave minimization problems. Acta Mathematica Vietnamica 8, 3-34.

[21] Tuy, H. (1987), Convex programs with an additional reverse convex constraint. Journal of Optimization Theory and Applications 52, 463-486..

[22] Tuy, H. and N.V. Thuong (1984), A finite algorithm for solving linear programs with an additional reverse convex constraint. Lecture Notes in Economics and Mathematical Systems 255, 291-304.

[23] Tuy, H. and N.V. Thuong (1985), Minimizing a convex function over the 
complement of a convex set. Methods of Operations Research 49, 85-89.

[24] [!y, H. and R. Horst (1988), Convergence and restart in branch-andbund aleorithms for global optimization. Applicarion to concave minimization and d.c. optimization problems. Mathematical Programming 41, 161-184.

Address of the authors:

P.T. Thach

Institute of Mathematics

P.O.Box 631 , Bo Ho

Hanoi, Vietnam

Rainer E. Burkard

Mathematisches Institut

Technische Universität Graz

Kopernikusgasse 24

A-8010 Graz,Austria

Werner Oettli

Fakultät für Mathematik und Informatik

Universität Mannheim

Postfach 103462

D-6800 Mannheim 1, Germany 